\title{
Combined effects of melatonin and topical hypothermia on renal ischemia-reperfusion injury in rats ${ }^{1}$
}

\author{
Pablo Cambeses Souza', Emanuel Burck dos Santos", Guilherme Lang Mottall', Silvia Regina Bonalv,
} Pedro Guilherme Schaefer ${ }^{\mathrm{V}}$, Daniela Campagnolv", Tiago Bortolini ${ }^{\mathrm{VII}}$, Carlos Otávio Corso ${ }^{\mathrm{VIII}}$

'Fellow Master degree, Postgraduate Program in Medicine: Surgical Sciences, Universidade Federal do Rio Grande do Sul (UFRGS), Porto Alegre-RS, Brazil. Conception and design of the study; technical procedures; acquisition, interpretation and analysis of data; manuscript writing.

"MSc, PhD, Department of Urology, Hospital de Clínicas de Porto Alegre (HCPA), Brazil. Conception and design of the study, critical revision.

I"Fellow Master degree, Postgraduate Program in Medicine: Surgical Sciences, UFRGS, Porto Alegre-RS, Brazil. Acquisition and interpretation of data; technical procedures.

IVPhD, Laboratory of Experimental Gastroenterology and Hepatology, HCPA, Porto Alegre-RS, Brazil. Acquisition and interpretation of data, technical procedures.

${ }^{\vee} M D$, Department of Pathology, HCPA, Porto Alegre-RS, Brazil. Histopathological examinations, acquisition of data.

VIPhD, Animal Experimentation Unit, HCPA, Porto Alegre-RS, Brazil. Technical procedures.

VIIFellow Master degree, Postgraduate Program in Medicine: Surgical Sciences, UFRGS, Porto Alegre-RS, Brazil. Interpretation and analysis of data, manuscript writing.

VIIIMSc, PhD, Associate Professor of Surgery, Digestive Surgery Unit, HCPA, and Department of Surgery, UFRGS, Porto Alegre-RS, Brazil. Conception and design of the study, interpretation of data, manuscript writing, critical revision, final approval.

\section{Abstract}

Purpose: To evaluate whether their combination was more effective than either alone in decreasing renal damage due to ischemia/reperfusion (I/R) injury in rats.

Methods: Thirty-two Wistar rats were assigned to four groups. Following right nephrectomy, their left kidneys were subjected to warm ischemia (IR), cold ischemia ( $T H+I R)$, intraperitoneal injection of $10 \mathrm{mg} / \mathrm{kg}$ melatonin $(\mathrm{MEL}+\mathrm{IR})$, or injection of $10 \mathrm{mg} / \mathrm{kg}$ melatonin followed by cold ischemia ( $M E L+T H+I R)$. Eight randomly assigned right kidneys constituted the control group. After 240 min of reperfusion, left nephrectomy was performed for histopathological evaluation, lipid peroxidation, and measurement of antioxidant enzyme activity. Serum was collected to measure urea and creatinine concentrations.

Results: Histopathological damage induced by ischemia and reperfusion was more attenuated in the $M E L+T H+I R$ group than in the $M E L+I R$ and $T H+I R$ groups $(p<0.037)$. Superoxide dismutase activity was significantly higher $(p<0.029)$ and creatinine $(p<0.001)$ and urea $(p<0.001)$ concentrations were significantly lower in the $M E L+T H+I R$ group than in the $M E L+I R$ and $T H+I R$ groups.

Conclusion: The combination of melatonin (MEL) and topical hypothermia (TH) better protects against renal I/R injury than does MEL or TH alone.

Key words: Melatonin. Hypothermia. Reperfusion Injury. Oxidative Stress. Kidney. Rats. 


\section{Introduction}

Renal ischemia, the most common cause of acute kidney injury, occurs in many clinical settings, such as renal transplantation, partial nephrectomy, and cardiovascular surgery ${ }^{1}$ and accounts for high mortality rates in intensive care units (ICUs) ${ }^{2}$. Renal ischemia-reperfusion $(\mathrm{I} / \mathrm{R})$ injury increases antibody production, which can be harmful to renal allografts, and may explain the pathophysiology underlying the association between delayed graft function and long-term allograft failure ${ }^{3}$. Blood flow during the reperfusion phase of $I / R$ injury can produce oxygen free radicals, which promote lipid peroxidation and can cause tissue injury. Lipid membrane peroxidation and oxidative damage to proteins and DNA can result in apoptosis and cell death ${ }^{4}$. I/R injury may result from the downregulation of the antioxidants, such as catalase, superoxide dismutase, and glutathione peroxidase ${ }^{5}$. Thus, antioxidants and radical scavengers, which inhibit this pathway, can protect from I/R injury ${ }^{6}$.

Topical hypothermia (TH) has also been shown to protect against renal I/R injury and has been used in kidney preservation, especially during kidney transplantation. A reduction in the core temperature of the kidney to below $4^{\circ} \mathrm{C}$ reduces the metabolism and enzymatic activity of the majority of kidney cells to $5-8 \%$. Improvement in the preserving solutions that target harmful pathways during cold storage leads to better preservation of the organ quality for a longer period of time ${ }^{8}$. Despite these advantages, hypothermia also has undesirable side effects, including cell swelling, acidosis, altered enzyme activity, calcium accumulation, and production of reactive oxygen species (ROS) ${ }^{9}$.

Melatonin (N-acetyl-5-methoxytryptamine) is an important product of the pineal gland that acts as a regulator of sleep, immune function, and circadian rhythm. Furthermore, as an electron donor, melatonin (MEL) is a potent scavenger of ROS ${ }^{10,11}$. MEL has been shown to have important antioxidant and anti-inflammatory effects in situations related to oxidative stress and inflammation ${ }^{12-14}$. MEL has been found to reduce $I / R$ injury, activate antioxidant enzymes such as superoxide dismutase (SOD) and catalase (CAT), and reduce lipid peroxidation and apoptosis ${ }^{10,15}$.

The finding that both $\mathrm{TH}$ and $\mathrm{MEL}$ reduce I/R injury suggested that the two together may have synergistic effects. Thus, in this study, we evaluated whether TH and MEL alone or in combination, had greater benefit in terms of improving histopathological parameters, oxidative stress damage, antioxidant enzyme activity, and biochemical parameters after $\mathrm{I} / \mathrm{R}$ in rat kidneys.

\section{- Methods}

The study was approved by the Animals Research Ethics Committee of Hospital de Clínicas de Porto Alegre (HCPA) (case 15-0472) and was performed in accordance with the International Guiding Principles for Biomedical Research Involving Animals, published by the Council for International Organizations of Medical Sciences (CIOMS), as well as with the Brazilian law on the scientific use of animals (Law 11794/2008).

\section{Animals and experimental design}

Adult male Wistar rats, weighing 276$406 \mathrm{~g}$ (age, 8-10 weeks old), were housed in the Animal Experimentation Unit of Hospital de Clínicas de Porto Alegre in groups of four in separate cages at room temperature $\left(22 \pm 2^{\circ} \mathrm{C}\right)$ with a 12-hour light/dark cycle and free access to water and rat chow. The rats were randomly allocated to four experimental groups of eight rats each. Following right nephrectomy, their left kidneys were subjected to warm ischemia 
for $40 \mathrm{~min}$ and reperfusion for $4 \mathrm{~h}$ (IR group); cold ischemia for $40 \mathrm{~min}$ and reperfusion for 4 h (TH+IR group); intraperitoneal injection of 10 $\mathrm{mg} / \mathrm{kg}$ MEL 10 min prior to warm ischemia for $40 \mathrm{~min}$ and reperfusion for $4 \mathrm{~h}$ (MEL+IR group); or intraperitoneal injection of $10 \mathrm{mg} / \mathrm{kg} \mathrm{MEL}$ $10 \mathrm{~min}$ prior to cold ischemia for $40 \mathrm{~min}$ and reperfusion for $4 \mathrm{~h}$ (MEL+TH+IR group). Eight randomly assigned right kidneys constituted the control group.

The surgical procedure began with the induction of inhalation general anesthesia with an isoflurane vaporizer at 3-5\%, administrated through a campanula. Blood was collected from the retro-orbital plexus. The animals were placed on a warm surgical table in the supine position, and their rear paw reflexes were tested to ensure that adequate anesthesia was achieved. Anesthesia was maintained with $1 \mathrm{~L} / \mathrm{min}$ oxygen and $2-3 \%$ isoflurane. A common electronic rectal thermometer (Termomed 1.0, Incoterm, Porto Alegre, Brazil) was used to assess systemic temperature. If needed, a heating lamp was used for maintaining the systemic temperature between $35.5^{\circ} \mathrm{C}$ and $37.5^{\circ} \mathrm{C}$. Before making an abdominal incision, bupivacaine $0.5 \%$ was injected into the abdominal wall to ensure pain control during and after the procedure. MEL (Sigma, St. Louis, MO, USA) was dissolved in absolute ethanol and diluted in saline to yield an ethanol concentration of $1 \%$. Rats in the $M E L+I R$ and $M E L+T H+I R$ groups were intraperitoneally administered MEL $(10 \mathrm{mg} /$ $\mathrm{kg}) 10 \mathrm{~min}$ prior to ischemia. A longitudinal median incision was made on the abdomen and surgical retractors were applied. Right nephrectomy was performed. Half of the right kidney was immersed in $10 \%$ formalin and the other half frozen and stored at $-80^{\circ} \mathrm{C}$. Ischemia was induced in the left kidneys by clamping the renal pedicle with an atraumatic microvascular surgery clip (Medicon, Tuttlingen, Germany). Cortical left kidney temperature was assessed with an intra-parenchymal probe connected to a specific thermometer (BAT 12, IITC Life Science, W. Hills, CA, USA). The left kidneys in the $T H, T H+I R$ and $M E L+T H+I R$ groups were flushed with ice-cold saline solution to achieve a target temperature of $4^{\circ} \mathrm{C}$. To avoid systemic hypothermia, a suction system was installed to remove the cold saline solution, and the left kidney was isolated from the rest of the abdominal cavity using a device made of polystyrene and latex. After 40 min of TH or warm ischemia, the clamp was removed from the renal pedicle and the abdominal wall was closed. The animals were moved to a new warm cage with water, but without food. After $4 \mathrm{~h}$ of reperfusion, they were moved again to the surgical table and anesthetized by inhalation to induce general anesthesia. The abdominal wall sutures were removed and left nephrectomy was performed. Blood samples were collected through heart puncture. Cardiectomy was performed to ensure animal death under anesthesia.

\section{Histopathological changes}

Half of each kidney was fixed in $10 \%$ formalin, embedded in paraffin, sliced, and stained with hematoxylin and eosin (H\&E) and periodic acid-Schiff (PAS) stain. The sections were examined under light microscope (Olympus bx41, Tokyo, Japan) at a magnification of $400 \times$ in a blinded manner by an experienced renal pathologist. The samples were graded histopathologically, as described previously ${ }^{16}$. Briefly, grade 1 indicated mitoses and necrosis of individual cells; grade 2 indicated necrosis of all cells in adjacent proximal convoluted tubules, with survival of surrounding tubules; grade 3 indicated necrosis confined to the distal third of the proximal convoluted tubule with a band of necrosis extending across the inner cortex; and grade 4 indicated necrosis affecting all three segments of the proximal convoluted tubules. Finally, normal findings were designated grade 0 (Figure 1). 


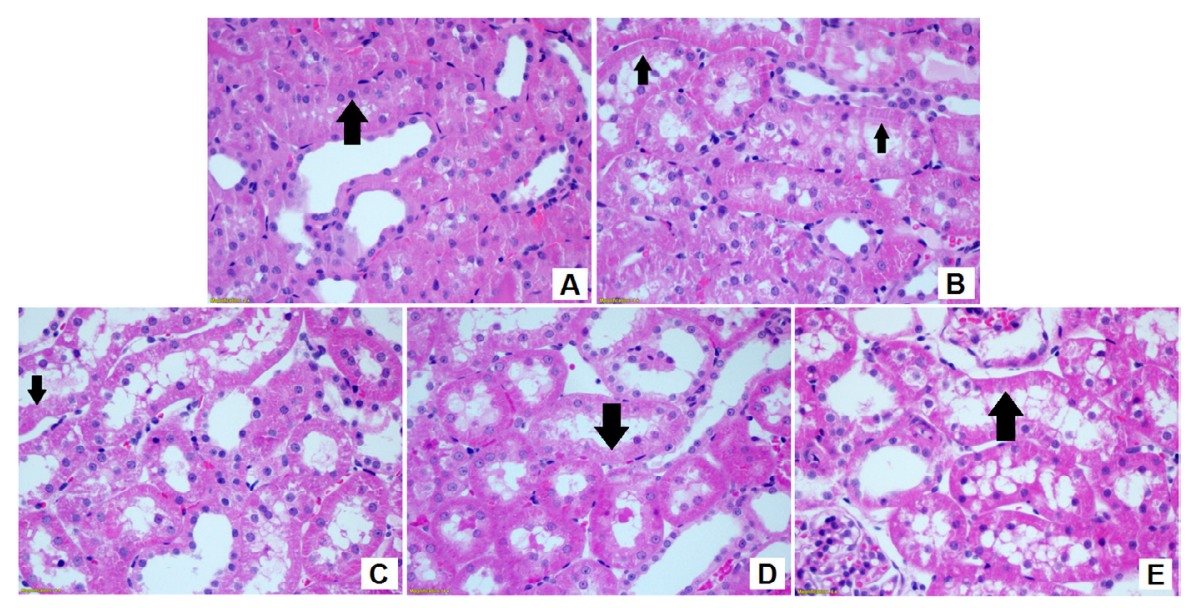

Figure 1 - Histopathological evaluation of rat kidneys showing the degrees of acute tubular necrosis described by Jablonski et al. ${ }^{16}$ (examined under a light microscope; Olympus bx41, Tokyo, Japan): Photomicrographs of the renal cortex: (A) showing no abnormality, grade 0 ( $\mathrm{HE} \times 400)$; (B) showing necrosis of individual cells and mitotic cells in Grade 1 acute tubular necrosis ( $\mathrm{HE} \times 400)$; (C) showing necrosis of a group of proximal convoluted tubules in Grade 2 acute tubular necrosis (HE $\times 400$ ); (D) showing necrosis of the distal third of the proximal convoluted tubules in Grade 3 acute tubular necrosis ( $\mathrm{HE} \times 400$ ); and (E) showing necrosis affecting all three segments of the proximal convoluted tubules.

Kidney homogenate preparation

Kidneys were weighed and homogenized for $40 \mathrm{sec}$ in an Ultra-Turrax homogenizer (IKA Works Inc., Wilmington, DE, USA) at $4^{\circ} \mathrm{C}$ in the presence of $1.15 \% \mathrm{KCl}(9 \mathrm{ml}$ per $\mathrm{g}$ of tissue) and phenyl-methyl-sulfonyl fluoride (PMSF) at a concentration of $100 \mathrm{mM}$ in isopropanol $(10 \mu \mathrm{l} / \mathrm{ml} \mathrm{KCl})$. The homogenate was centrifuged for $10 \mathrm{~min}$ at 3,000 rpm in a refrigerated centrifuge (SORVALL Super T21; Kendro Laboratory Products, Weaverville, NC, USA), the precipitate was discarded, and the supernatant was frozen at $-80^{\circ} \mathrm{C}$ for subsequent biochemical analysis.

\section{Protein}

Protein concentrations were determined using the Bradford method with bovine albumin as a standard, followed by spectrophotometry at $595 \mathrm{~nm}$.

\section{Oxidative stress damage}

Malondialdehyde (MDA) levels were measured using thiobarbituric acid reactive substances (TBARS).

\section{Antioxidant enzyme activity}

Superoxide dismutase (SOD) activity was determined by assessing the inhibition of superoxide-dependent adrenaline autooxidation, spectrophotometrically at $480 \mathrm{~nm}$. The results were expressed as USOD/min/mg protein. Catalase (CAT) activity was calculated based on the decomposition of hydrogen peroxide, as shown spectrophotometrically at $240 \mathrm{~nm}$, with the results expressed as pmol/ mg protein.

\section{Biochemical functional parameter analysis}

Serum concentrations of urea and creatinine $(\mathrm{sCr})$ at baseline and at the end of the 
experiment, from the blood samples collected from the retro-orbital plexus and heart, respectively, were estimated using a Roche Cobas 8000 c702 automatic biochemistry apparatus. Biochemical functional parameters were analyzed only with regard to experimental groups because urea and $\mathrm{sCr}$ were estimated from peripheral blood.

\section{Statistical analysis}

Data were presented as frequency and percentage or as mean \pm SD. Associations between variables were assessed using $\chi^{2}$ tests. Continuous variables were compared by one-ANOVA, followed by Turkey's test for post hoc comparisons. Statistical significance was set at $p<0.05$. All statistical analyses were performed using the Statistical Package for the Social Sciences (PASW Statistics for Windows, Version 18.0. Chicago: SPSS Inc).

\section{Results}

The baseline characteristics of rats in the four study groups and the control group, including weight, temperature, and serum creatinine and urea concentrations, were similar (data not shown).

Histopathological evaluation revealed significant differences among the groups studied (Figure 2). The degree of I/R injury was greater in the IR, TH+IR and MEL+IR groups than in the control group. There are rats in the IR and TH+IR groups presented with grade 4 injury, whereas none of the rats in the MEL+IR group presented with a grade 4 injury, and one had a grade 0 injury. The $M E L+T H+I R$ group showed the lowest degree of injury, with none of the rats having a grade 3 or 4 injury and one having grade 0 , similar to the control group of kidneys not subjected to I/R.

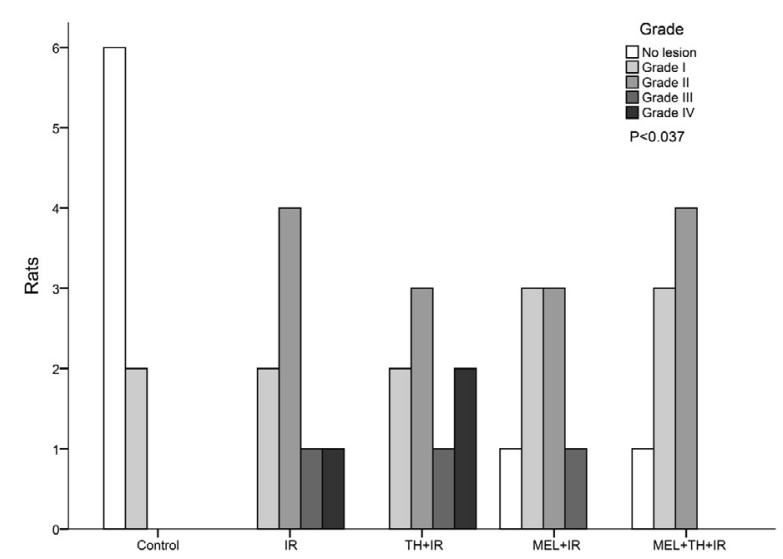

Figure 2 - Differences in histopathological grade between the groups. IR, ischemia and reperfusion; $\mathrm{TH}+\mathrm{IR}$, topical hypothermia + ischemia and reperfusion; MEL+IR, melatonin + ischemia and reperfusion; MEL+TH+IR, melatonin + topical hypothermia + ischemia and reperfusion. Chisquared test $(p<0.037)$.

Oxidative stress damage was measured by TBARS tissue assay, which assesses the degree of lipid peroxidation. TBARS values were highest in the IR and TH+IR groups, but were lower in rats administered MEL. The TBARS values were significantly lower in the $M E L+I R$ than in all of the other groups $(p<0.05$; Figure 3).

SOD activity was lower in the IR than in the other groups, and was significantly higher in the $M E L+T H+I R$ group than in the IR group $(p<0.029), C A T$ release was higher in the $\mathrm{MEL}+\mathrm{TH}+\mathrm{IR}$ group than in the other groups, but the difference was not statistically significant $(p>0.05)$. Assessment of functional parameters at the end of the experimental protocol showed that serum creatinine and urea concentrations were highest in the IR group. The concentrations of both were significantly lower in the $M E L+T H+I R$ group than in the IR and $M E L+I R$ groups $(p<0.05)$. Differences from baseline were also lowest in the $M E L+T H+I R$ group. The $T H+I R$ group showed significant differences in creatinine concentrations compared with the IR and MEL+IR groups (Figures 4 and 5). 


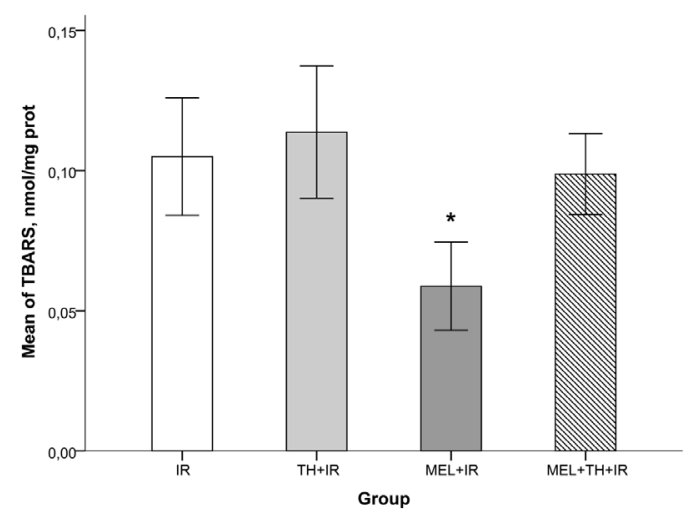

Figure 3 - Means of TBARS. IR, ischemia and reperfusion; TH+IR, topical hypothermia + ischemia and reperfusion; MEL+IR, melatonin + ischemia and reperfusion; $M E L+T H+I R$, melatonin + topical hypothermia + ischemia and reperfusion; TBARS, thiobarbituric acid reactive species. ${ }^{*} M E L+I R$ vs $I R, p<0.002 ; T H+I R$, $p<0.001 ; \mathrm{MEL}+\mathrm{TH}+\mathrm{IR}, \mathrm{p}<0.008$. Note: For comparing continuous variables the one-ANOVA test was used. Turkey's test was applied for post-hoc comparisons.
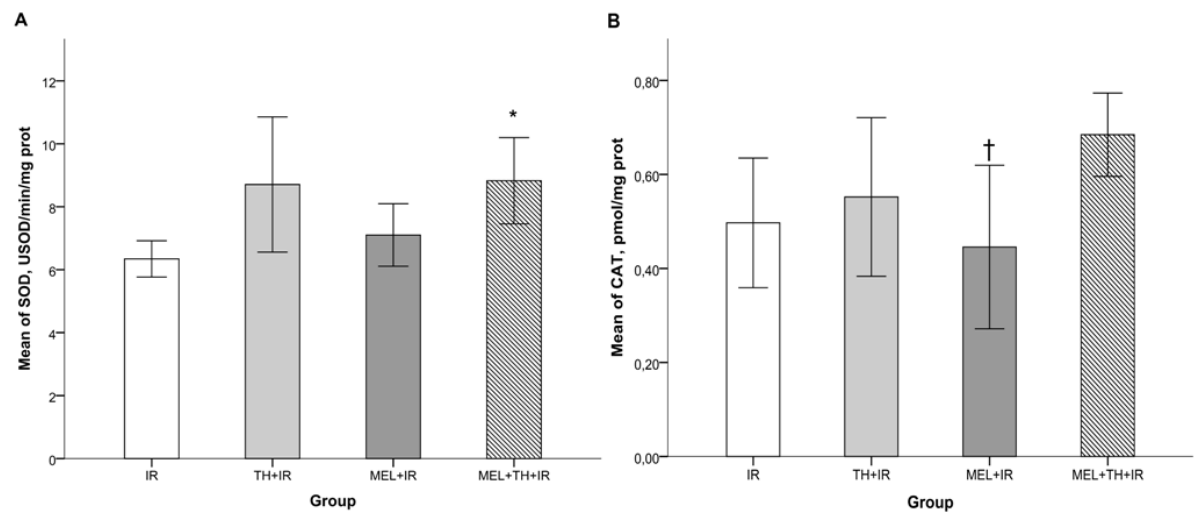

Figure 4 - Means of SOD and Catalase. IR, ischemia and reperfusion; TH+IR, topical hypothermia + ischemia and reperfusion; $M E L+I R$, melatonin + ischemia and reperfusion; $M E L+T H+I R$, melatonin + topical hypothermia + ischemia and reperfusion; SOD, superoxide dismutase. A, SOD; $B$, catalase. ${ }^{*} I R$ vs. MEL+TH+IR ( $\left.p<0.029\right)$. ${ }^{\dagger}$ No statistically significant differences. Note: For comparing continuous variables, a one-ANOVA test was used. Turkey's test was applied for post-hoc comparisons.
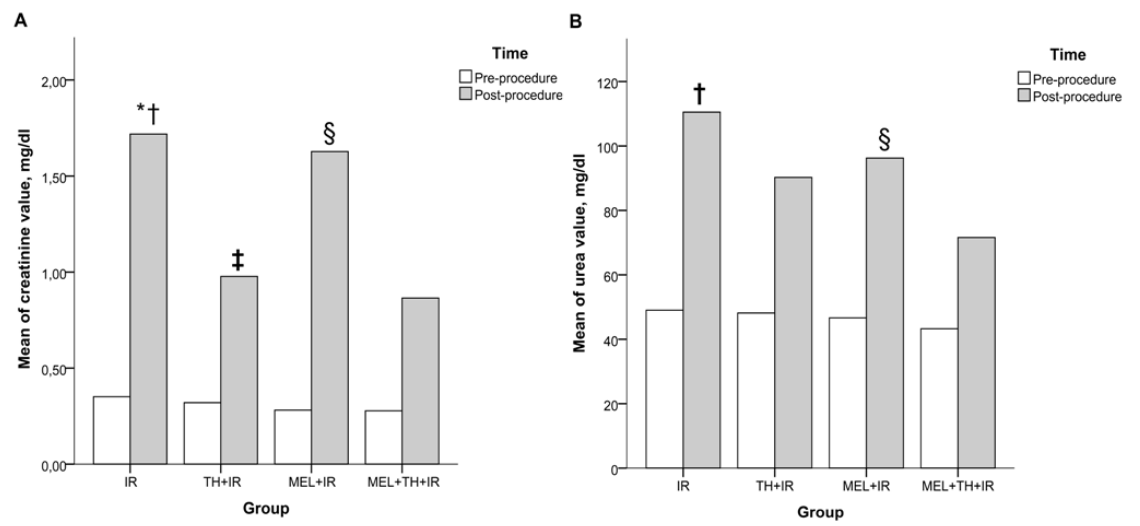

Figure 5 - Means of creatinine and urea levels between the study groups before and after the procedure. $I R$, ischemia and reperfusion; $T H+I R$, topical hypothermia + ischemia and reperfusion; $M E L+I R$, melatonin + ischemia and reperfusion; MEL+TH+IR, melatonin + topical hypothermia + ischemia and reperfusion. $A$, creatinine; $B$, urea. ${ }^{*} I R$ vs $T H+I R(p<0.001) ;{ }^{\dagger} I R$ vs. $M E L+T H+I R(p<0.001) .{ }^{\ddagger} T H+I R$ vs. $M E L+I R(p=0.001)$. ${ }^{\S} \mathrm{MEL}+\mathrm{IR}$ vs $\mathrm{MEL}+\mathrm{TH}+\mathrm{IR}(\mathrm{p}=0.001)$. Note: For comparing continuous variables the two-way ANOVA test was used. Turkey's test was applied for post-hoc comparisons. 


\section{- Discussion}

In our study, I/R induced histopathological changes, including grades 3 and 4 injury ${ }^{16}$, in both the IR and TH+IR groups. Pre-treatment with MEL alone (MEL+IR) slightly attenuated these histopathological damages, with no kidney experiencing grade 4 injury, but the damage did not differ significantly from that in the control group. MEL has been shown to act as a cytoprotective agent in I/Rinduced lesions, reversing the damage caused by $\mathrm{I} / \mathrm{R}$ nephrotoxicity ${ }^{17}$. The combination of MEL pre-treatment plus topical hypothermia at $4^{\circ} \mathrm{C}(\mathrm{MEL}+\mathrm{TH}+\mathrm{IR})$ markedly reduced the degree of histopathological damage induced by I/R. Although some of these kidneys had grade 1 and 2 injuries, none had grade 3 and 4 lesions, thereby exhibited no difference with the control group. Thus, combined treatment showed benefits, reducing the histopathological extent of tissue injury.

Several studies have evaluated the effects of MEL alone or in combination with other substances on I/R injury ${ }^{18-20}$. Indeed, one previous study evaluated whether pretreatment with $\mathrm{MEL}$ reduced I/R injury ${ }^{21}$. In that study, which used an experimental IR model in rats, pre-treatment with $10 \mathrm{mg} / \mathrm{kg}$ MEL 10 minutes before ischemia attenuated histopathological changes and reduced biochemical indices. I/R-induced renal damage can also be reduced by topical hypothermia, which reduces cellular metabolism and oxidative stress, making it essential for organ viability during the period of ischemia ${ }^{7}$.

I/R-induced renal injury is associated with lipid peroxidation, a mechanism that causes oxidative damage to the cell membrane, leading to the production of free radicals ${ }^{10}$, and TBARS is a good indicator of the degree of lipid peroxidation. We found that the level of TBARS was significantly higher in the IR group, indicative of increased lipid peroxidation due to high oxidative stress ${ }^{22}$. We also found that TBARS was significantly lower in the MEL+IR group than in the other groups $(p<0.001)$. This finding, that MEL reduces the production of TBARS, indicates that MEL decreases lipid peroxidation and cellular damage. This reduction in lipid peroxidation may be partly due to MEL scavenging the peroxynitrite (ONOO-) radicals and hydroxyl $(\mathrm{OH})$ ions $^{23}$. Although TBARS also tended to be lower in the $M E L+T H+I R$ group than in the IR and IR+TH groups, these differences were not significant.

I/R causes accumulation of free radicals and the reduction of antioxidant enzymes, which have deleterious effects on the cell membrane, DNA, and protein. SOD and CAT activities decrease markedly during renal ischemia, with the main factor being the time of exposure to ischemic insult ${ }^{24,25}$. In agreement with findings showing that MEL activates SOD ${ }^{10}$, we found that SOD activity was significantly higher in the $M E L+T H+I R$ group than in the IR group $(p<0.029)$. In contrast, neither $\mathrm{TH}$ nor MEL alone significantly increased SOD activity. These findings suggest that the combination of MEL and TH stimulated protective antioxidant enzymes, corroborating our histopathological findings.

We also found that IR significantly increased creatinine and urea concentrations, showing that $I / R$ had deleterious effects on renal function, even acutely. Creatinine concentrations were lower in the $M E L+T H+I R$ than in the IR and MEL+IR groups, showing that combined therapy additionally protects renal function $(p<0.001)$. Moreover, cold ischemia alone in the TH+IR group showed better protection of renal function when compared with the IR and MEL+IR groups $(p<0.001)$. As expected, post-ischemia urea concentrations were higher in the IR group, showing that $\mathrm{I} / \mathrm{R}$ damaged renal function. Although urea concentrations were lower in the $\mathrm{TH}+\mathrm{IR}$ and $M E L+I R$ groups than in the IR group, the 
differences were not statistically significant. In contrast, urea concentrations were significantly lower in the $\mathrm{MEL}+\mathrm{TH}+\mathrm{IR}$ group than in the other groups $(P<0.001)$, likely due to the synergistic protective effect of both interventions. The benefits of cold ischemia at $4^{\circ} \mathrm{C}$ include reduction of cellular metabolism ${ }^{26,27}$ and in the production of xanthine oxidase ${ }^{7}$, whereas the benefits of MEL include the activation of antioxidant enzymes and the protection of oxidative phosphorylation. Thus, together, cold ischemia and MEL reduce creatinine and urea concentrations, both parameters of renal function.

To our knowledge, our study is the first to assess the effects of both MEL and TH on IRinduced renal injury. This experimental model was found to be adequate, as IR-induced histopathological changes, altered factors related to oxidative stress, and worsened renal function. All of these parameters were attenuated by MEL and TH, especially by their combination. This combination showed benefits in the main outcome studied, histopathology, as well as altering the activity of the antioxidant enzyme SOD, and the functional parameters creatinine and urea.

Although MEL has been studied in several contexts, its role in organ transplantation has not yet been established. Experimental studies have shown, however, that MEL may have potential beneficial effects on organ transplantation ${ }^{28}$. MEL has a wide therapeutic window and few important side effects ${ }^{29}$, suggesting that further studies are warranted.

In addition to $\mathrm{TH}$, current clinical strategies to reduce kidney damage by $I / R$ include perfusion fluids, use of a hypothermic perfusion machine, administration of mannitol, and a reduced period of ischemia ${ }^{30}$. The use of MEL, if proven effective in animal models of $\mathrm{I} / \mathrm{R}$ and in humans, may become a particularly helpful tool in preventing I/R-induced kidney damage.

\section{Conclusion}

The combination of melatonin (MEL) and topical hypothermia (TH) was more effective at attenuating ischemia-reperfusion induced injury than was either alone.

\section{References}

1. Paller MS. Acute renal failure: controversies, clinical trials, and future directions. Semin Nephrol. 1998;18(5):482-9. PMID: 9754600.

2. Rodriguez F, Bonacasa B, Fenoy FJ, Salom MG. Reactive oxygen and nitrogen species in the renal ischemia/reperfusion injury. Curr Pharm Des. 2013;19(15):2776-94. PMID: 23092323.

3. Fuquay R, Renner B, Kulik L, McCullough JW, Amura C, Strassheim D, Pelanda R, Torres R, Thurman JM. Renal ischemiareperfusion injury amplifies the humoral immune response. J Am Soc Nephrol. 2013;24(7):1063-72. PMID: 23641055.

4. Kosieradzki $M$, Rowinski W. Ischemia/ reperfusion injury in kidney transplantation: mechanismsand prevention. TransplantProc. 2008;40(10):3279-88. PMID:19100373.

5. Singh I, Gulati S, Orak JK, Singh AK. Expression of antioxidant enzymes in rat kidney during ischemia-reperfusion injury. Mol Cell Biochem. 1993;125(2):97-104. PMID: 8283974.

6. Greenwald RA. Superoxide dismutase and catalase as therapeutic agents for human diseases. A critical review. Free Radic Biol Med. 1990;8(2):201-9. PMID: 2185145.

7. Southard JH, Belzer FO. Organ preservation. Annu Rev Med. 1995;46:235-47. PMID: 7598460.

8. McAnulty JF, Reid TW, Waller KR, Murphy CJ. Successful six-day kidney preservation using trophic factor supplemented media and simple cold storage. Am J Transplant. 2002;2(8):712-8. PMID: 12243492.

9. Bartels-Stringer $M$, Kramers $C$, Wetzels JF, Russel FG, Groot H, Rauen U. Hypothermia causes a marked injury to rat proximal tubular cells that is aggravated by all currently used preservation solutions. Cryobiology. 2003;47(1):82-91. PMID: 12963415. 
10.Reiter RJ, Tan DX, Osuna C, Gitto E. Actions of melatonin in the reduction of oxidative stress. A review. J Biomed Sci. 2000;7(6):44458. PMID: 11060493.

11.Reiter RJ, Tan DX, Terron MP, Flores LJ, Czarnocki Z. Melatonin and its metabolites: new findings regarding their production and their radical scavenging actions. Acta Biochim Pol. 2007;54(1):1-9. PMID: 17351668.

12.Carrillo-Vico A, Guerrero JM, Lardone PJ, Reiter RJ. A review of the multiple actions of melatonin on the immune system. Endocrine. 2005;27(2):189-200. PMID: 16217132.

13. Mayo JC, Sainz RM, Tan DX, Hardeland $\mathrm{R}$, Leon J, Rodriguez C, Reiter RJ. Antiinflammatory actions of melatonin and its metabolites, N1-acetyl-N2-formyl-5methoxykynuramine (AFMK) and N1acetyl-5-methoxykynuramine (AMK), in macrophages. J Neuroimmunol. 2005;165(12):139-49. PMID: 15975667.

14. Reiter RJ, Tan DX, Maldonado MD. Melatonin as an antioxidant: physiology versus pharmacology. J Pineal Res. 2005;39(2):2156. PMID: 16098101.

15. Reiter RJ, Guerrero JM, Garcia JJ, Acuna-Castroviejo D. Reactive oxygen intermediates, molecular damage, and aging. Relation to melatonin. Ann N Y Acad Sci. 1998;854:410-24. PMID: 9928448.

16.Jablonski P, Howden BO, Rae DA, Birrell CS, Marshall VC, Tange J. An experimental model for assessment of renal recovery from warm ischemia. Transplantation. 1983;35(3):198204. PMID: 6340272.

17.Banaei S, Ahmadiasl N, Alihemmati A. Comparison of the protective effects of erythropoietin and melatonin on renal ischemia-reperfusion injury. Trauma Mon. 2016;21(3):e23005. PMID: 27921018.

18.Yilmaz M, Mogulkoc $R$, Baltaci AK. Effect of three-week zinc and melatonin supplementation on the oxidant-antioxidant system in experimental renal ischemiareperfusion in rats. Acta Clin Croat. 2015;54(4):395-401. PMID: 27017711.

19.Sezgin G, Ozturk G, Guney S, Sinanoglu O, Tuncdemir M. Protective effect of melatonin and 1,25-dihydroxyvitamin D3 on renal ischemia-reperfusion injury in rats. Ren Fail. 2013;35(3):374-9. PMID: 23356461.
20.Kurcer Z, Oguz E, Ozbilge H, Baba F, Aksoy $\mathrm{N}$, Celik H, Cakir H, Gezen MR. Melatonin protects from ischemia/reperfusioninduced renal injury in rats: this effect is not mediated by proinflammatory cytokines. J Pineal Res. 2007;43(2):172-8. PMID: 17645695.

21.Sener G, Sehirli AO, Keyer-Uysal M, Arbak $S$, Ersoy $Y$, Yegen BC. The protective effect of melatonin on renal ischemia-reperfusion injury in the rat. J Pineal Res. 2002;32(2):1206. PMID: 12071469.

22. Eschwege $P$, Paradis $V$, Conti $M$, Holstege $A$, Richet $F$, Deteve J, Menager $P$, Legrand A, Jardin A, Bedossa P, Benoit G. In situ detection of lipid peroxidation by-products as markers of renal ischemia injuries in rat kidneys. J Urol. 1999;162(2):553-7. PMID: 10411087.

23. Reiter RJ, Oh CS, Fujimori O. Melatonin Its intracellular and genomic actions. Trends Endocrinol Metab. 1996;7(1):22-7. PMID: 18406721.

24.Li Z, Nickkholgh A, Yi X, Bruns H, Gross ML, Hoffmann K, Mohr E, Zorn M, Buchler MW, Schemmer P. Melatonin protects kidney grafts from ischemia/reperfusion injury through inhibition of NF-kB and apoptosis after experimental kidney transplantation. J Pineal Res. 2009;46(4):365-72. PMID: 19552759.

25.Conti $M$, Eschwege $P$, Ahmed $M$, Paradis $V$, Droupy S, Loric S, Bedossa P, Charpentier B, Legrand A, Benoit G. Antioxidant enzymatic activities and renal warm ischemia: correlation with the duration of ischemia. Transplant Proc. 2000;32(8):2740-1. PMID: 11134780.

26.Santos EB, Koff WJ, Grezzana Filho Tde J, De Rossi SD, Treis L, Bona SR, Pegas KL, Katz B, Meyer FS, Marroni NA, Corso CO. Oxidative stress evaluation of ischemia and reperfusion in kidneys under various degrees of hypothermia in rats. Acta Cir Bras. 2013;28(8):568-73. PMID: 23896835.

27. Ribeiro GB, Santos EBD, Bona SR, Schaefer PG, Garcez TA, Rabolini EB, Smaniotto GP, Marroni NP, Corso CO. The effects of local ischemic preconditioning and topical hypothermia in renal ischemia/reperfusion injury in rats. Acta Cir Bras. 2017;32(10):81626. PMID: 29160368.

28.Esteban-Zubero E, Garcia-Gil FA, Lopez- 
Pingarron $L$, Alatorre-Jimenez $M A$, Inigo-Gil P, Tan DX, Garcia JJ, Reiter RJ. Potential benefits of melatonin in organ transplantation: a review. J Endocrinol. 2016;229(3):R129-46. PMID: 27068700.

29.Andersen LP, Gogenur I, Rosenberg J, Reiter RJ. The Safety of melatonin in humans. Clin Drug Investig. 2016;36(3):169-75. PMID: 26692007.

30.Spaliviero M, Power NE, Murray KS, Sjoberg $\mathrm{DD}$, Benfante NE, Bernstein ML, Wren J, Russo P, Coleman JA. Intravenous mannitol versus placebo during partial nephrectomy in patients with normal kidney function: a double-blind, clinically-integrated, randomized trial. Eur Urol. 2018;73(1):53-9. PMID: 28822586.

\section{- Acknowledgements}

To the team of the Animal Experimentation Unit (UEA), Experimental Pathology Unit, Department of Clinical Pathology, Laboratory of Experimental Hepatology and Physiology, and Molecular and Protein Analysis Unit (UAMP) of Hospital de Clínicas de Porto Alegre (HCPA) for their assistance and technical support.

\section{Correspondence:}

Carlos Otávio Corso

Disciplina de Cirurgia Digestiva, HCPA

Rua Ramiro Barcelos, 2350/sala 747

90035-903 Porto Alegre - RS Brasil

Tel.: (55 51)3359-8232

corso@portoweb.com.br

Received: Nov 27, 2017

Review: Jan 26, 2018

Accepted: Feb 28, 2018
Conflict of interest: none

Financial sources: FIPE/HCPA
${ }^{1}$ Research performed at Animal Experimentation Unit, Hospital de Clínicas de Porto Alegre (HCPA), Brazil. Part of Master degree thesis, Postgraduate Program in Medicine: Surgical Sciences. Tutor: Carlos Otávio Corso. 